Un champ de luttes et de forces : l'espace des prises de position sur la gouvernance dans la crise de l'euro (2010-2016)

Frédéric Lebaron

\author{
(2) OpenEdition \\ Journals \\ Édition électronique \\ URL : http://journals.openedition.org/ei/6349 \\ DOI : 10.4000/ei.6349 \\ ISSN : 2553-1891 \\ Éditeur
}

Association Économie et Institutions

Référence électronique

Frédéric Lebaron, « Un champ de luttes et de forces : l'espace des prises de position sur la gouvernance dans la crise de l'euro (2010-2016) », Économie et institutions [En ligne], 27 | 2018, mis en ligne le 20 novembre 2018, consulté le 20 avril 2019. URL : http://journals.openedition.org/ei/6349 DOI : $10.4000 /$ ei.6349

Ce document a été généré automatiquement le 20 avril 2019.

Revue Économie et institutions 


\title{
Un champ de luttes et de forces : l'espace des prises de position sur la gouvernance dans la crise de l'euro (2010-2016)
}

\author{
Frédéric Lebaron
}

1 Dans un ouvrage intitulé La double démocratie. Une Europe politique pour la croissance, publié en janvier 2017, Michel Aglietta et Nicolas Leron, économistes régulationnistes d'orientation social-démocrate, «développe[nt] des propositions concrètes pour surmonter la crise européenne en commençant par rendre aux citoyens le pouvoir de se prononcer sur les grandes options économiques» (présentation de l'éditeur, Aglietta \& Leron 2017).

2 Cette prise de position n'est que l'une des plus récentes d'un ensemble de déclarations, appels, contributions, discours, etc., émanant d'acteurs de toutes sortes, qui ont pour point commun de partir d'un constat de crise de/au sein de la zone euro ${ }^{1}$, et de se présenter comme des réponses plus ou moins cohérentes à cette crise, fondées sur des arguments d'économie politique plus ou moins longuement développés.

3 Cet article vise à établir la pertinence d'une analyse des enjeux traversés par la zone euro depuis décembre 2009 (qui est la date de la dégradation de la note financière de la dette grecque par trois agences de notation) à partir de la notion de champ telle que Pierre Bourdieu l'a développée (Bourdieu 1992), en partant ici non des positions et caractéristiques des agents chargés de la "gouvernance économique » de la zone, mais des prises de positions qui se sont multipliées dès les premières attaques spéculatives sur les titres de la dette publique grecque, qui constituent aussi ce que les analystes du discours ont appelé un « champ discursif » (Maingueneau 1983) dont l'analyse est en ellemême pertinente (pour de premiers éléments : Lebaron 2016).

4 Un premier enjeu de la notion de champ consiste à en délimiter les frontières, qui sont toujours un enjeu de luttes symboliques. On s'attache donc ici à une définition (1/) et un recensement (2/) à visée systématique - sinon exhaustive - des prises de position des 
acteurs concernés ${ }^{2}$ durant la période étudiée. Ce recensement ne peut être que partiel tant il touche très largement non seulement des acteurs européens, mais aussi mondiaux, à différentes échelles. On déterminera ainsi par le même mouvement un corpus de textes, de natures diverses, qui constituera le matériau qualitatif principal de cet article, tout en étant aussi son premier « résultat de recherche » à proprement parler.

La troisième partie, en s'appuyant sur le même corpus de textes, discute diverses typologies couramment proposées, souvent par les différents acteurs eux-mêmes ${ }^{3}$, en vue de décrire l'espace des prises de position sur la crise. Le travail classificatoire spontané des agents fait en effet partie des luttes dans le champ et son étude permet ainsi de préciser la description des prises de position en jeu ${ }^{4}$.

en conclusion, on examinera brièvement la question de la dynamique des prises de position durant la période 2010-2016, qui correspond à la période de crise de l'euro la plus intense, entre fin 2009-début 2010 et juillet 2012, et à une phase plus modérée caractérisée par le débat sur les politiques d'austérité.

\section{Qu'est-ce qu'une prise de position?}

$7 \quad$ L'une des difficultés associées au choix de mener l'analyse à partir des prises de position est le fait que celles-ci doivent préalablement être définies et délimitées, alors même que la notion de prise de position renvoie, d'une manière générale, à des raisonnements, analyses, arguments, énoncés souvent de nature complexe et multidimensionnelle, se traduisant par des pratiques discursives qui peuvent s'objectiver sous diverses formes : publication de livres, rapports, interviews, communiqués, textes de blogs, tweets, conférences, affirmations dans des débats à la radio ou télévisés, etc. La difficulté du « codage » des prises de position a souvent été soulignée dans les travaux prosopographiques (par exemple, Sapiro 1996), et l'on peut penser que la difficulté est devenue encore plus grande à l'heure du numérique, avec la multiplication des supports possibles d'expression.

8 On se penche ici sur les prises de position relatives à l'avenir de la zone dans le contexte de ladite " crise ", commencée en décembre 2009. On exclut donc a priori, par exemple, des prises de position qui seraient limitées à un seul pays (la Grèce, l'Allemagne ou la France), même si la distinction entre prises de position sur la zone dans sa globalité et prises de position restreintes à un pays est parfois très difficile à mettre en œuvre concrètement, dans la mesure où la crise dans un pays peut apparaitre comme un test pour la zone dans son ensemble (comme ce fut justement le cas pour la Grèce à partir de 2010), ou encore lorsque la taille et l'importance des pays leur confèrent immédiatement un retentissement européen (Allemagne et France notamment).

Prendre position suppose qu'un "problème public », ou au moins un "enjeu », soit préalablement défini ou construit, suscitant précisément un ensemble de discours spécifiques, ce qui ne va jamais complètement de soi (Hassenteufel 2011). Pour la crise de la zone euro, c'est le cas à partir de la fin de 2009, lorsque la spéculation avivée sur la dette publique grecque soulève un questionnement plus global pour l'avenir de la monnaie unique (voir Picard [ed.], 2015) ${ }^{5}$. L'accroissement des écarts entre les taux d'intérêt (ce que la plupart des commentateurs, même francophones, nomment aussi les spread) sur les dettes souveraines nationales s'accompagne en effet de divergences importantes des niveaux d'endettement public, en particulier à la suite de la crise des 
subprimes et des politiques menées pour y faire face, lesquelles ont également dû répondre à des effets négatifs de la récession variables selon les pays. Ce contexte macroéconomique particulier justifie à la fois une mobilisation discursive et institutionnelle portant sur le cas grec, puis les autres pays menacés (Chypre, Irlande, Portugal, Espagne, et, par contagion, Italie et France), mais aussi, plus largement, des inquiétudes sur la soutenabilité des dettes souveraines et, dès lors, sur l'avenir même de la zone euro.

10 L'existence-même de l'euro étant récente, l'apparition d'un enjeu discursif centré sur l'avenir économico-institutionnel de la zone euro n'est en réalité que la transformation postérieure à la crise des subprimes, donc la reconfiguration, d'un espace de débat préexistant, qui s'est en particulier structuré lors des négociations sur l'Union économique et monétaire (UEM) et a fortement contraint l'expression sur le sujet. Cet espace prédéfini constitue un arrière-plan discursif permanent à l'ensemble du débat économique et monétaire européen. L'une des difficultés que l'on rencontre ici est que, comme tout secteur de politique publique, l'enjeu de l'articulation entre la politique monétaire et la politique budgétaire en zone euro peut être décrit comme un champ de luttes discursives permanentes, celles-ci évoluant au fil du temps en fonction de nombreux facteurs, et que dans ce champ des raisonnements, arguments, analyses plus ou moins sophistiqués ne cessent d'être mobilisés et de se confronter les uns aux autres dans ce qui peut apparaître de loin comme une cacophonie, au sein de laquelle les acteurs mettent en œuvre diverses stratégies en fonction de leurs positions et des contextes.

\section{L'espace des prises de position sur l'avenir de la zone euro : essais de construction}

11 Les prises de position, dans un tel espace multidimensionnel et mouvant, ne se structurent donc que sur la base d'un ensemble de contraintes préexistantes, qui forment l'environnement au sein duquel elles se déploient. En particulier, ce que l'on peut appeler l'orthodoxie institutionnelle, s'agissant de la zone euro (sa définition, son fonctionnement, etc.), est inscrite à tout moment dans un cadre juridique contraignant, en l'occurrence celui des traités dérivés du traité de Maastricht (voir annexe $1 /$ texte 1$)^{6}$. Elle définit une forme de doxa, de fait et de droit, dans le champ. Il faut entendre ici doxa dans un sens fort : il s'agit d'un référent juridique surplombant et polarisant très fortement l'ensemble des débats au sein de la zone.

12 Une spécificité des champs de politique publique est, rappelons-le, la centralité d'un référent juridique: dans le cas de la zone euro, c'est le texte-même des traités qui constitue ce qui est aussi, à de nombreux égards, un discours constituant (Maingueneau \& Cossuta 1995), c'est-à-dire un discours dont l'autorité fondatrice est sous-jacente à d'autres discours, qui n'ont besoin que de s'y référer, fût-ce de façon allusive, pour en mobiliser l'autorité propre.

Les prises de position s'échelonnent donc grossièrement, des plus légitimistes (celles, en particulier, formées par les discours officiels des acteurs et institutions centraux euxmêmes) aux plus déviantes, celles qui sont produites et diffusées par un ensemble très vaste d'acteurs à différents niveaux et dans différents pays. La configuration des prises de position peut donc être représentée, en première approximation, par un "centre " doxique et une périphérie au sein de laquelle des prises de position hétérogènes ont pour 
point commun de s'opposer à des degrés variables à cette doxa. L'ensemble des luttes autour de la production des discours et donc des décisions officielles peut dès lors être décrit comme relevant d'un champ restreint, espace en grande partie institutionnel, qui est inscrit dans le champ plus global de la gouvernance européenne (Georgakakis \& Lebaron 2014).

L'espace des prises de position dans sa globalité définit quant à lui le champ élargi, un espace sans limite prédéfinie : un texte de blog rédigé par un économiste américain peut, à certaines conditions, constituer une intervention efficiente dans ce champ (pour un exemple, le blog de Paul Krugman sur le site du New York Times, qui évoque très fréquemment, surtout après 2010, l'enjeu dans la zone euro: cf. annexe 9/texte 9). Inversement, une micro-organisation politique centrée sur la lutte contre l'euro ou l'Union européenne peut n'être que très faiblement efficiente dans le champ, ce qui revient à dire largement « négligeable » du point de vue de l'analyse sociologique globale de la dynamique du champ (comme, par exemple, le parti politique Union populaire république de François Asselineau, qui s'est défini autour de l'enjeu européen).

\subsection{Le retour au " pacte de stabilité et de croissance » originel comme stratégie de sortie de crise : une orthodoxie juridico-institutionnelle et sa formulation radicale}

15 Fin 2009, le débat conjoncturel au sein des États européens est, comme dans d'autres pays, de plus en plus centré sur les stratégies de sortie de crise (en anglais : exit strategies) L'année 2009 a été marquée par une récession très profonde, la mise en place de "plans de relance " plus ou moins importants dans différentes régions du monde et la dégradation du niveau d'endettement public dans de nombreux pays.

Dès la mi-2009, des voix se font entendre au sein de la Banque centrale européenne sur la nécessité de rétablir très rapidement la situation « normale », i.e. conforme au « cadre des Traités " selon une formule fréquemment utilisée, y compris au sein des institutions européennes (voir Lebaron 2016). Elles s'expriment durant toute la période cruciale du basculement relativement rapide vers les politiques de désendettement par le rétablissement de l'équilibre budgétaire, qui vont être qualifiées par leurs opposants de " politiques d'austérité » (cf. annexe 5/texte 5).

17 Les traités définissent en effet un «cadre » doctrinal et discursif - institutionnel relativement étroit, puisque, d'une part, la banque centrale a pour mission exclusive la stabilité des prix et que, d'autre part, des règles budgétaires strictes (règle des $3 \%$ de déficit budgétaire, des $60 \%$ de dette publique par rapport au PIB) doivent permettre de garantir le respect par les États d'une politique compatible avec le cadre commun ( annexe $2 /$ texte 2 ).

18 L'adhésion à ce cadre, qui peut être plus ou moins distanciée et agrémentée de propositions de réforme ou d'évolution, définit ainsi une première famille de prises de position, qui, en un sens, ne fait que réactiver, après une période relativement incertaine perçue comme une parenthèse, le fonctionnement «normal » ou « usuel » de l'UEM tel qu'il est inscrit dans les traités. La Commission en déploie la forme la plus institutionnelle, qui a également pris corps chez les acteurs politiques, allemands puis européens (cf. textes $n^{\circ} 1$ et $\left.n^{\circ} 2\right)^{7}$.

19 La nouveauté de la période qui s'ouvre fin 2009 tient au fait que l'affirmation de la nécessité d'un retour à ce cadre s'accompagne, chez un certain nombre d'acteurs, de l'expression d'une insatisfaction face à l'absence de garanties institutionnelles 
concernant l'application des textes, les États-membres étant suspectés de ne pas s'y plier dès qu'il leur est possible de le faire, comme cela a été le cas à diverses reprises. On voit ainsi rapidement émerger l'idée que le cadre juridique actuel est insuffisant dans ses conditions d'application et doit être complété par de nouveaux dispositifs, lesquels vont rapidement s'incarner dans le Traité sur la Surveillance, le Contrôle et la Gouvernance (ratifié en 2012, soit peu de temps après le début de la crise grecque), suscitant une floraison d'innovations et de discours politico-institutionnels allant dans le même sens, bien au-delà du champ restreint (annexe 1/texte 1).

20 À l'intérieur de cet espace de l'orthodoxie juridico-institutionnelle, se construit ainsi, avec un certain succès dans un premier temps, puisqu'elle aboutit à des modifications substantielles des traités, une position plus radicale : en résumé, il s'agit de renforcer les mécanismes d'intégration européenne entendus essentiellement (ce qui est bien sûr une définition de l'intégration) comme un ensemble de règles, dispositifs de surveillance et sanctions. On pourrait bien sûr qualifier cette interprétation en référence à l'ordolibéralisme (on aurait une forme plus ou moins radicale de celui-ci) ou à l'opposition classique dans le domaine monétaire entre faucons et colombes, dogmatiques et pragmatiques, etc. Il faudrait alors, comme le fait Paul Krugman, transférer cette catégorisation initialement réservée à la monnaie, aux enjeux de politique budgétaire qui l'accompagnent dans le cas de la zone euro.

21 Il suffit à ce stade de décrire ce sous-espace de prises de position comme celui, relativement flou si l'on considère ses frontières, de l'orthodoxie des traités, laquelle, divisée entre des modalités plus ou moins radicales, tend globalement à devenir plus stricte et effective avec les réactions face à la crise de la zone euro ${ }^{8}$, suscitant en retour un surcroît de prises de position opposées, de natures assez diverses, en premier lieu opposées à l'euro.

\subsection{Les « anti-euro »}

À l'opposé strict de l'orthodoxie juridico-institutionnelle, on sait que depuis sa création, l'euro a suscité le fort scepticisme d'un grand nombre d'acteurs et commentateurs au sein et en dehors de la zone euro, lesquels ont, notamment, argué que la zone monétaire concernée n'était pas optimale, que les hétérogénéités économiques, commerciales, voire culturelles, entre les pays qui la composent, condamnaient ce projet utopique à plus ou moins brève échéance, notamment du fait de l'asymétrie entre une politique monétaire, donc une politique ou du moins un taux de change, conçues et mises en œuvre à l'échelle de la zone, et des politiques budgétaires nationales. Plusieurs auteurs annoncent ainsi régulièrement l'effondrement de la zone euro, dans un discours que l'on peut qualifier de prophétique et à visée performative (cf. annexe $3 /$ texte 3$)^{9}$ : en affirmant sans cesse la mort inéluctable de l'euro, on contribue bien sûr à mettre en cause toute confiance dans la monnaie unique. De même que l'orthodoxie institutionnelle se traduit par des discours qui font exister l'euro en fondant sa naturalité, les discours hostiles à l'euro tendent ainsi, logiquement, à insister sur l'aberration quasiment « contre-nature » que constituerait la zone euro (en son état actuel tout au moins) à divers égards.

Certains des économistes les plus prestigieux au niveau international se sont donc divisés depuis l'origine sur le sujet, mais il convient plus largement de rappeler que l'hostilité à l'euro comme monnaie unique constitue un pôle structurant du champ et que, depuis 2009, ce pôle, qui constitue aussi un sous-espace différencié, a semble-t-il plutôt renforcé 
sa présence dans l'espace public, sous diverses formes, même si le travail de quantification en la matière est difficile. On le voit par exemple avec la publication en 2016 d'un ouvrage du « Nobel » Joseph Stiglitz, qui dénonce le rôle de l'euro dans la crise européenne (Stiglitz 2016). Parmi le "grand public», la légitimité de l'euro a subi également une certaine érosion, au moins durant une partie de la période, même si les évolutions ne sont en fait pas très marquées.

Encadré 1. Le soutien à la monnaie unique (France, Allemagne) dans les enquêtes eurobaromètres

Les eurobaromètres indiquent les tendances de « l'opinion » s'agissant de la monnaie unique. En 1995, la proportion de personnes hostiles à l'idée de monnaie unique en France n'était que de $10 \%, 19 \%$ des personnes interrogées n'ayant pas d'avis ou ne répondant pas. Juste après la crise des subprimes, le soutien à la monnaie unique se situe à $70 \%$ en novembre 2010 (les personnes « contre » représentent $26 \%$ ). En novembre 2011, le rapport s'établit à $63 \%$ contre $32 \%$, et évolue à $62 \%$ contre $33 \%$ en mai 2013. En mai 2016, le rapport est revenu à $69 \%$ contre $24 \%$. Durant la période 2010-2016, les variations sont relativement importantes, avec une période de dégradation marquée puis un rétablissement relatif aux niveaux élevés de l'avantcrise. On observe des variations similaires dans le cas allemand, à des niveaux assez proches. L'enquête eurobaromètre spécifique d'octobre 2016 confirme le rétablissement relatif du soutien à l'euro, avec $56 \%$ au sein de la zone. Parallèlement, le soutien aux institutions de la zone (la BCE) connaît lui-aussi une très forte baisse, suivie d'un léger rétablissement.

La crise de la zone euro a fourni beaucoup de matière à la mobilisation "anti-euro " depuis fin 2009, avec des arguments puisés à diverses sources et le cas échéant hétérogènes, allant, schématiquement, de l'anti-capitalisme à l'ultra-libéralisme, en passant par le souverainisme ou le nationalisme économiques. Une partie de ces arguments met ainsi en avant l'asymétrie de situation entre l'Allemagne, qui bénéficie commercialement d'un euro relativement faible, et les pays du Sud qui sont au contraire mis en difficulté par le niveau trop élevé de l'euro pour leurs économies. L'argument commercial «structurel » s'accompagne le cas échéant d'arguments relatifs à l'absence de caractère démocratique du pilotage de la zone euro par la $\mathrm{BCE}$, institution de nature avant tout technocratique, et aux règles budgétaires contraignantes, arguments sur lesquels le débat s'est fréquemment cristallisé.

On peut ainsi, là encore, parler d'un sous-espace des prises de position « anti-euro », qui vont du rejet plus global de toute construction européenne à un scepticisme plus ou moins fort sur la capacité de la zone euro à trouver un fonctionnement collectivement satisfaisant, et aspirent à d'autres possibilités d'organisation (monnaie commune, système monétaire européen, etc.).

\subsection{Refondations institutionnelles et politiques}

Le troisième sous-espace de prises de position a, semble-t-il, connu lui-aussi depuis 2009 une forte croissance, en relation avec les différentes phases de la crise, la mise en œuvre et les conséquences des politiques d'austérité, et bien sûr avec la montée en puissance de l'opposition à l'euro. Il s'agit de l'ensemble des contributions prônant, dès 2010, un changement plus ou moins radical dans l'organisation institutionnelle de la zone euro, 
cela afin de la rendre susceptible de résister à divers "chocs » ou crises à l'avenir, et allant à des degrés variables dans un sens différent de (voire clairement opposé à) celui de l'orthodoxie institutionnelle.

On peut ici rapidement distinguer deux types de prises de position au sein de cet espace : certaines sont compatibles avec la totalité ou une grande partie des traités actuels, mais elles modifient de façon incrémentale tel ou tel aspect de la gouvernance économique et monétaire de la zone (cf. ici l'exemple de la « modeste proposition » de Yanis Varoufakis, annexe $4 /$ texte 4 , qui se situait dans la perspective d'une inflexion pragmatique et progressive dans le cadre des traités, donc sans lourde machinerie institutionnelle, et s'appuyait sur une typologie fine des propositions débattues à différents niveaux, développée dans le texte 5 et le texte 6); d'autres supposent une refonte plus ou moins importante, voire une "refondation" complète, des traités actuels et sont dès lors marqués par leur caractère juridique.

Un ensemble de propositions qui a émergé durant la crise concerne essentiellement la capacité de la zone euro à mener une politique budgétaire commune dans un contexte de forte hétérogénéité. Est surtout visée ici l'asymétrie entre l'intégration fédérale de la politique monétaire et la persistance d'un système décentralisé : en mettant en place divers dispositifs tels que l'union bancaire, des " eurobonds ", voire un budget de la zone euro plus important, il s'agit de construire les conditions d'une politique budgétaire d'ensemble de la zone qui fasse en quelque sorte contrepoids à la politique monétaire centralisée. Cette perspective, classiquement associée aux positions françaises, se situe à la frontière de l'orthodoxie institutionnelle, qui se nourrit d'ailleurs de certaines de ces idées, face aux constats réitérés et assez largement partagés de l'insuffisance et de l'asymétrie de la construction initiale.

Ces propositions «techniques» (mais aux lourdes conséquences politiques potentielles) sont souvent accompagnées de propositions de refonte de la gouvernance d'ensemble de la zone, en général dans un sens plus " démocratique » : c'est le cas par exemple de l'idée, développée dans le manifeste pour une union politique de l'euro lancé en février 2014, de mettre en place un Parlement de la zone euro, idée portée par Thomas Piketty (annexe 7/ texte 7), ou, moins radicale, l'idée d'un ministre des Finances de la zone, etc. Ces dernières supposent une négociation internationale et, sans aucun doute, une refonte au moins partielle des traités.

Des propositions plus radicales, supposant une refonte plus importante des traités, donc une négociation beaucoup plus complexe, circulent également, comme par exemple l'articulation entre "plan A » et "plan B », lancée autour de Jean-Luc Mélenchon, Oskar Lafontaine, etc., qui mobilise la menace d'une sortie de la zone en cas d'échec de ces négociations (annexe 8/texte 8). Le vocabulaire de la "refondation de la zone euro » renvoie ainsi à des actes plus solennels et institutionnellement efficients. Le mot "refondation» indique une mise en cause de la sacralité du discours juridique constituant, à savoir le cadre des traités.

\subsection{Les « non-prises de position »}

31 De nombreux acteurs ne prennent pas position sur la question de l'euro et il est important de rappeler que l'espace des acteurs qui prennent position dans un enjeu de débat public ne constitue jamais un échantillon représentatif de la population, ou même d'une sous-population particulière (les économistes, les hommes politiques, etc.): 
prendre position, c'est déjà s'affirmer comme légitime et mobilisé par le sujet, ce qui ne concerne que des individus aux caractéristiques particulières.

\section{Typologies, catégorisations et qualifications mutuelles}

32 La classification opérée au 1. Qu'est-ce qu'une prise de position?) se veut avant tout descriptive et analytique. Dans la pratique, les auteurs ne cessent de se classer euxmêmes et de classer les autres en utilisant divers procédés discursifs, ce qui contribue à la dynamique des relations entre les trois sous-espaces étudiés, dans un mouvement de lutte perpétuelle. On en donnera une série d'exemples, en complément de ceux que nous avons déjà évoqués (comme la transposition, tentée notamment par Krugman, de l'opposition faucon/colombe aux questions budgétaires-institutionnelles).

Les propositions développées par James Galbraith et Yanis Varoufakis pour l'ensemble de la zone euro, surtout à partir de 2010, sont qualifiées par eux-mêmes de «modest proposal » ("proposition modeste»), en raison du fait qu'elles ne nécessitent pas de lourde modification des traités, mais peuvent être mises en œuvre à partir des institutions existantes avec un minimum de volonté politique (textes 4 à 6), et « dynamiser » l'économie de la zone euro par une action budgétaire spécifique. Elles sont explicitement opposées à un lourd travail de refonte ou refondation institutionnelle de la zone, et situées dans deux grands ensembles d'orientations : les unes "austéritaires » ( austerians), les autres qualifiées de « camp modeste » (modest camp, voir annexe 5/texte 5).

Les partisans du démantèlement de la zone euro décrivent fréquemment l'ensemble des autres économistes ou acteurs publics comme persévérant dans le déni des défauts rédhibitoires et des « contre-performances » de la zone euro, comme on le voit bien avec les analyses de Jacques Sapir (annexe 3/texte 3), à l'image de nombreux discours oppositionnels. L'utilisation de catégories psychologiques et psychanalytiques est bien sûr courante pour caractériser ses opposants, mais elle est ici mobilisée pour rendre compte de la persistance de l'euro malgré des constats qui devraient conduire à son dépassement.

Les partisans d'un démantèlement de la zone euro, qualifiés d' "anti-euros ", sont euxmêmes fréquemment disqualifiés comme "anti-européens", "nationalistes", « populistes », « souverainistes », etc., par les défenseurs de la perspective de l'euro, qu'ils soient fondamentalistes ou pragmatiques, attachés ou non aux règles des traités, etc. Dans la logique classificatoire spontanée qui caractérise la vie politique, les positions relatives à la monnaie ou au budget sont ainsi souvent agrégées avec des prises de position d'autres natures, par exemple sur les politiques migratoires. Il est vrai que, dans beaucoup de pays, les positions hostiles à l'euro apparaissent souvent portées par des défenseurs de politiques hostiles à l'immigration.

Les prises de position relevant de la catégorie 2.3. (Refondations institutionnelles et politiques) mobilisent fréquemment l'idée d'un dépassement de l'opposition entre la continuation des politiques et institutions actuelles et le «repli» nationaliste qu'impliquerait la simple sortie de la zone. On le voit bien avec Yanis Varoufakis et son parti politique DIEM25, créée en 2015 (textes 4 à 6). Ils contribuent ainsi à la formation d'une structure ternaire, constituée par trois types de prises de position dont nous avons vu qu'ils relevaient de deux champs emboîtés (champ restreint et champ élargi): 
attachement plus ou moins radical aux règles des traités, fortement encastré dans le champ restreint/fin de l'euro, le plus souvent portée par des acteurs périphériques / dépassement dans de nouvelles politiques et de nouvelles institutions, souvent lié à des positions d'entre-deux, entre champ restreint et champ élargi.

Enfin, il est fréquent que cette troisième catégorie de prise de position soit stigmatisée comme incohérente ou utopique par l'un ou l'autre des deux autres groupes de prises de position, engagés dans une lutte farouche: comme toute prise de position « intermédiaire », celle-ci est jugée ambivalente, instable ou tout simplement intenable, faute d'une nécessaire clarification relative à l'avenir de l'euro en tant que monnaie.

\section{Conclusion : quels processus évolutifs?}

En dépit des évolutions importantes qu'a connu la zone euro depuis 2010 (création du FESF et du MES, TSCG, union bancaire), cet espace de prises de position apparait relativement stable. À quelques exceptions près, les déplacements d'acteurs ont été relativement lents et peu marqués depuis 2010: il est extrêmement rare qu'une institution, un mouvement ou un individu soit passé d'un soutien radical aux règles des traités au rejet de celles-ci et réciproquement. On a cependant vu proliférer les prises de position, notamment au sein de la catégorie 2.3., et des micro-déplacements à l'intérieur de chaque sous-catégorie. Tout déplacement majeur est précédé de déplacements plus microscopiques, un peu comme dans un processus d'ébullition ${ }^{10}$.

Dans chaque sous-espace pris isolément, des mouvements plus fins sont en effet repérables. Les prises de position visant à un durcissement des règles, qui s'étaient multipliées après 2010 dans les gouvernements et les institutions européennes, ont eu tendance à être fragilisées à partir de 2013, comme l'illustre le rapport des cinq présidents de 2015 (texte X), relativement modéré et avançant quelques éléments qui le rapprochent plus clairement de la catégorie 2.3. À l'opposé, des organisations se sont rapprochées de positions plus hostiles à l'euro ou exigeant des changements institutionnels plus radicaux.

40 En revanche, les rapports de force politiques et sociaux entre ces prises de position se sont quant à eux fortement modifiés. Les organisations politiques prônant la sortie (nationale) de l'euro ont plutôt eu tendance à progresser et l'audience des économistes " anti-euro » à s'accroître, selon divers indicateurs qu'il conviendrait de préciser (on pense au succès du blog de Jacques Sapir, par exemple), parallèlement à une érosion du soutien à l'euro dans l'opinion, qui a été stoppée, en France, après 2014. De même, la multiplication des "propositions» de réforme a nourri un courant de réflexion sur le changement institutionnel qui est devenu très important dans certains pays, comme la France, ainsi que l'illustrent l'ouvrage d'Aglietta et Leron ou encore les propositions de Thomas Piketty, reprises dans le contexte électoral par le candidat socialiste Benoît Hamon. À l'opposé, le contexte de campagne électoral en Allemagne peut conduire à un renouveau des propositions radicales de renforcement des règles des traités, comme l'illustrent des prises de position récentes de Wolfgang Schäuble, qui visent précisément à contrer la montée en puissance de l'hostilité à l'euro en Allemagne, sous l'égide de l'Alternative für Deutschland.

41 Il restera à comprendre comment se structurent les prises de position dans le champ, et comment s'interprète cette dynamique: d'une part, au sein du champ restreint, qui 
correspond au pôle de la gouvernance économique européenne, et d'autre part au sein du champ élargi, les deux, fortement interdépendants, ne cessant bien sûr d'interagir.

\section{BIBLIOGRAPHIE}

Aglietta M. \& N. Leron (2017), La double démocratie. Une Europe politique de la croissance, Paris, Le Seuil.

Bourdieu P. (1992), Les règles de l'art. Genèse et structure du champ littéraire, Paris, Le Seuil.

Campbell John L. \& Ove K. Pedersen (2001), The Rise of Neoliberalism and Institutional Analysis, Princeton and Oxford, Princeton University Press.

Économie et Institutions, $\mathrm{n}^{\mathrm{o}} 22,1^{\mathrm{er}}$ semestre 2015, Varia ; https://journals.openedition.org/ei/970

Eurobaromètre : http://ec.europa.eu/COMMFrontOffice/publicopinion/index.cfm

Georgakakis D. \& F. Lebaron (2014), « Administrative and political economic elites in Europe:

economics as a dominant resource?», communication lors du colloque international

"Understanding the Transformations of Economic Elites in Europe », Université de Lausanne, 18-20 juin.

Gusfield J. (2009), La culture des problèmes publics. L'alcool au volant : la production d'un ordre symbolique, Paris, Éditions Economica.

Hassenteufel P. (2011), Sociologie politique : l'action publique, Paris, Armand Colin.

Lebaron F. (2016), « Zone euro : la crise de la doxa des politiques d'austérité », Idées économiques et sociales, $\mathrm{n}^{\circ} 183$, mars, p. 39-46.

Maingueneau D. (1983), Sémantique de la polémique, Lausanne, l'Âge d'Homme.

Maingueneau D. \& F. Cossutta (1995), «L'analyse des discours constituants », Langages, $29^{\mathrm{e}}$ année, $\mathrm{n}^{\circ} 117$.

Picard Robert G. (2015), The Euro Crisis in the Media. Journalistic coverage of economic crisis and European institutions, university of Oxford/Reuters, Institute for the Study of Journalism.

Sapiro G. (1996), « La raison littéraire. Le champ littéraire français sous l'occupation. 1940-1944 », Actes de la Recherche en Sciences Sociales, Année 1996, vol. 111, nº 1, p. 3-35.

Stiglitz J. (2016), L'euro : comment la monnaie unique menace l'avenir de l'Europe, Paris, Les Liens qui Libèrent.

\section{NOTES}

1. Nous conservons pour l'instant le flou relatif de la notion de «crise » dans le cas qui nous occupe, car chaque prise de position est aussi une prise de position sur l'existence ou non d'une ou plusieurs crises et sur la nature de celle-ci.

2. Il s'agit également de délimiter l'espace des acteurs pertinents. 
3. On a en particulier bénéficié du travail de classification réalisé par Yanis Varoufakis dans le texte 5 en annexe 5 .

4. Un test empirique de cette construction sera effectué dans un prochain travail à partir des programmes des candidats à l'élection présidentielle 2017.

5. En fait, les interrogations sur l'avenir de l'euro s'intensifient entre début 2010 et l'acmé de mai 2010 (avec le lancement du Securities Market Program), puis ne cessent de rebondir jusqu'à nos jours.

6. "Simulation results suggest that even with average annual consolidation efforts of $0.5 \%$ of GDP, returning to the pre-crisis euro area debt ratio would take around two decades. Therefore, to safeguard government solvency and sustainable fiscal positions in the euro area countries, governments must give fiscal consolidation top priority, taking the ongoing recovery of the economy into account, and bring their deficit ratios to below $3 \%$ of GDP, in line with the recommendations by the Ecofin Council. Consolidation efforts will have to last for several years in many countries. In Europe, the Stability and Growth Pact provides the appropriate framework for the coordination of the necessary consolidation policies. It is now crucial that all governments strictly adhere to their commitments under the Pact. Supporting national fiscal frameworks should be strengthened wherever necessary" (annexe 1/texte 1).

7. "The global economic and financial crises have exposed shortcomings in the governance of the Economic and Monetary Union. A reform package - the so-called Six Pack - will enter into force by the end of the year. It amends and strengthens the Stability and Growth Pact (SGP), introduces a new Excessive Imbalances Procedure and lays down new requirements for Member States' national budgetary frameworks. While it will be a game changer, more should be done specifically for the euro area. Today, the Commission is unveiling two new Regulations aimed at further strengthening the surveillance mechanisms in the euro area, as well as a Green Paper on presenting options for euro Stability Bonds" (annexe 2/ texte 2).

8. Il faut évidemment distinguer le durcissement des règles budgétaires, avec le TSCG notamment, et la création du FESF puis du MES (embryon de fonds monétaire européen) et d'une union bancaire en 2014, qui vont dans le sens d'un élargissement de la gamme des actions publiques possibles et peut être associé à une moindre orthodoxie monétaire.

9. "C'est l'une des raisons pour lesquelles les nombreuses unions monétaires qui ont été constituées ont très souvent échoué. Les processus de dissociation monétaire ont été en réalité bien plus importants que les processus d'unification monétaire. L'échec de la zone euro est en réalité "normal" au regard de l'histoire. Il est donc clair, aujourd'hui, que l'éventualité d'une explosion de la zone euro fait partie des scénarii sur lesquelles les Banques Centrales des pays membres travaillent. » (annexe 3/texte 3)

10. Voir sur ces différents points le numéro 22 d'Économie et Institutions, $1^{\mathrm{er}}$ semestre 2015, consacré au discours et le numéro $23,2^{\mathrm{e}}$ semestre 2015 , consacré à la crise de l'euro.

\section{RÉSUMÉS}

Sur la base de l'étude qualitative d'un corpus de textes en anglais et en français, on analyse les prises de position sur la gouvernance dans la zone euro durant la période 2010-2016 : elles dessinent un espace multidimensionnel structuré autour de pôles opposés, mais définissant un continuum d'orientations. Se structurant les unes en fonction des autres, elles doivent être conçues comme les éléments d'un rapport de force. 
La définition de la notion de prise de position fait l'objet d'une première partie, suivie d'un « essai de construction » de l'espace sur une base qualitative. On distingue ainsi l'orthodoxie juridico-institutionnelle, les prises de position anti-euro et diverses tentatives de refondations institutionnelles et politiques, auxquelles s'ajoutent les «non-prises de position». On conclut ensuite sur les dynamiques évolutives durant la séquence historique étudiée.

On the basis of a qualitative corpus analysis (texts in French and English), we analyse the position-takings about Euro area governance during the period 2010-2016. It allows to draw a multidimensional space structured by opposite poles, in a continuum of orientations. These orientations structure themselves in relation to each-other in a set of power relations.

The first part consists in an attempt to define the notion of position-taking, before we try to construct the space of position-takings on a qualitative basis. We distinguish between a juridicoinstitutional orthodoxy, anti-euro stances and a stance of attempts to rebuild the euro area politically and institutionally. To these position-takings one must add the "no-position-taking". We then assess the evolutionary dynamics of this space.

\section{INDEX}

Keywords : position-taking, juridico-institutional orthodoxy, euro, Euro area, governance Code JEL E60 - General, E65 - Studies of Particular Policy Episodes, N44 - Europe: 1913-

\section{AUTEUR}

\section{FRÉDÉRIC LEBARON}

Professeur de sociologie, École normale supérieure Paris-Saclay, Institutions et dynamiques historiques de l'économie et de la société (IDHES) 